\title{
The crystal structure of Ac-AChBP in complex with $\alpha$-conotoxin LvIA reveals the mechanism of its selectivity towards different $\mathrm{nAChR}$ subtypes
}

\author{
Manyu $\mathrm{Xu}^{1}$, Xiaopeng $\mathrm{Zhu}^{2}$, Jinfang $\mathrm{Yu}^{1}$, Jinpeng $\mathrm{Yu}^{2}$, Sulan Luo ${ }^{2 \bowtie}$, Xinquan Wang ${ }^{1 凶}$ \\ ${ }^{1}$ The Ministry of Education Key Laboratory of Protein Science, School of Life Sciences, Beijing Advanced Innovation Center \\ for Structural Biology, Collaborative Innovation Center for Biotherapy, Tsinghua University, Beijing 100084, China \\ ${ }^{2}$ Key Laboratory of Tropical Biological Resources, Ministry of Education, Key Lab for Marine Drugs of Haikou, Hainan \\ University, Haikou 570228, China \\ $\bowtie$ Correspondence: luosulan2003@163.com (S. Luo), xinquanwang@mail.tsinghua.edu.cn (X. Wang) \\ Received March 17, 2017 Accepted April 27, 2017
}

\begin{abstract}
The $\alpha 3^{*}$ nAChRs, which are considered to be promising drug targets for problems such as pain, addiction, cardiovascular function, cognitive disorders etc., are found throughout the central and peripheral nervous system. The $\alpha$-conotoxin ( $\alpha$-CTx) LvIA has been identified as the most selective inhibitor of $\alpha 3 \beta 2$ nAChRs known to date, and it can distinguish the $\alpha 3 \beta 2 \mathrm{nAChR}$ subtype from the $\alpha 6 / \alpha 3 \beta 2 \beta 3$ and $\alpha 3 \beta 4$ nAChR subtypes. However, the mechanism of its selectivity towards $\alpha 3 \beta 2, \alpha 6 / \alpha 3 \beta 2 \beta 3$, and $\alpha 3 \beta 4$ nAChRs remains elusive. Here we report the co-crystal structure of LvIA in complex with Aplysia californica acetylcholine binding protein (Ac-AChBP) at a resolution of $3.4 \mathrm{~A}$. Based on the structure of this complex, together with homology modeling based on other nAChR subtypes and binding affinity assays, we conclude that Asp-11 of LvIA plays an important role in the selectivity of LvIA towards $\alpha 3 \beta 2$ and $\alpha 3 / \alpha 6 \beta 2 \beta 3$ nAChRs by making a salt bridge with Lys- 155 of the rat a3 subunit. Asn-9 lies within a hydrophobic pocket that is formed by Met-36, Thr-59, and Phe-119 of the rat $\beta 2$ subunit in the $\alpha 3 \beta 2 \mathrm{nAChR}$ model, revealing the reason for its more potent selectivity towards the $\alpha 3 \beta 2 \mathrm{nAChR}$ subtype. These results provide molecular insights that
\end{abstract}

Manyu Xu and Xiaopeng Zhu contributed equally to this work.

Electronic supplementary material The online version of this article (doi:10.1007/s13238-017-0426-2) contains supplementary material, which is available to authorized users. can be used to design ligands that selectively target a3ß2 nAChRs, with significant implications for the design of new therapeutic $\alpha-C T x s$.

KEYWORDS base editor, high-fidelity, mouse embryos, proximal-site deamination, whole-genome sequencing

\section{INTRODUCTION}

Neuronal nicotinic acetylcholine receptors (nAChRs) are a group of ligand-gated cation-selective ion channels that play key roles in fast signal transmission in the nervous system (Zoli et al., 2015; Hurst et al., 2013). They are activated by neurotransmitters such as acetylcholine and choline, and they also respond to numerous non-endogenous neuroactive molecules such as nicotine (Cecchini and Changeux, 2015). The nAChRs are implicated in various neurological diseases, including pain, Alzheimer's and Parkinson's disease, substance addiction, epilepsy, attention deficit hyperactivity disorder, and depression (Dineley et al., 2015; Le Novere et al., 2002; Laviolette and van der Kooy, 2004), which makes them important drug development targets.

The nAChRs belong to the Cys-loop superfamily of pentameric ligand-gated ion channels ( $\mathrm{LLGIC}$ ), a superfamily which also includes serotonin (5-HT3), gamma-aminobutyric-acid (GABAA and GABAC), and glycine receptors (Ortells and Lunt, 1995). In vertebrates, neuronal nAChRs are composed of $\alpha$ subunits $(\alpha 2-\alpha 10)$ and $\beta$ subunits $(\beta 2-\beta 4)$, which combine to form a large array of homo- or hetero-pentamers, such as $(\alpha 3)_{3}(\beta 2)_{2},(\alpha 3)_{3}(\beta 4)_{2},(\alpha 7)_{5}$, et 
cetera (Le Novere et al., 2002; Karlin, 2002). Both $\alpha$ and $\beta$ subunits consist of an extracellular $\mathrm{N}$-terminal ligand-binding domain, four C-terminal transmembrane regions (M1-M4), and an intracellular region extending between M3 and M4 (Hendrickson et al., 2013). Due to sequence and structural homology, especially the high similarity in the ligand-binding site among different nAChRs, the development of novel drugs specific for one type of nAChR or addressing the specificity and selectivity issues of natural agonists and antagonists is a very challenging task, which requires the provision of detailed structural information.

Structural studies of $n A C h R s$ have experienced a number of important breakthroughs in the past decades (Karlin, 2002; Unwin, 1995; Brejc et al., 2001; Celie et al., 2004; Morales-Perez et al., 2016). The early results came from low-resolution cryo-EM studies of the Torpedo sp. acetylcholine (ACh) receptor (Unwin, 1993, 1995, 2005; Beroukhim and Unwin, 1995; Miyazawa et al., 1999). On the other hand, the crystal structure of the acetylcholine-binding proteins (AChBPs) from mollusks, which are soluble homologs of the extracellular domain (ECD) of nAChR (Brejc et al., 2001; Smit et al., 2001), represented a great leap in the understanding of the structure and function of nAChRs. After this breakthrough, the X-ray crystallographic structures of mouse muscle-type a1 (Dellisanti et al., 2007), two $\alpha 7$ nAChR ECD-AChBPs chimeras (Li et al., 2011; Nemecz and Taylor, 2011), human neuronal a9 nAChR ECD (Zouridakis et al., 2014), and the full-length heteromeric human $(\alpha 4)_{3}(\beta 2)_{2}$ receptor were determined in quick succession (Morales-Perez et al., 2016). In addition, crystal structures of AChBPs or nAChR ECD in complex with different types of ligands, including AChBPs/nicotine (Celie et al., 2004), AChBPs/a-cobratoxin (Bourne et al., 2005), AChBPs/aconotoxins (PnIA (A10L, D14K) (Celie et al., 2005), TxIA (A10L) (Dutertre et al., 2007), Iml (Hansen et al., 2005; Ulens et al., 2006), and GIC (Lin et al., 2016)), human a9 nAChR ECD/methyllycaconitine (Zouridakis et al., 2014), and human a2 nAChR ECD/epibatidine (Kouvatsos et al., 2016), provided valuable information for our understanding of receptorligand binding specificity and selectivity. Interestingly, despite the only $20 \%-24 \%$ sequence identity with nAChRs, AChBPs display a striking structural resemblance to the $\mathrm{nAChR} E C D$, and their pharmacological properties closely resemble those of nAChRs. Therefore, AChBPs still represent the best template for the characterization of ligand binding to the extracellular ligand-binding domain of nAChRs (Cecchini and Changeux, 2015).

Conotoxins are disulfide-bridged peptides isolated from cone-snail venom, which act on a wide range of ion channels, including voltage-gated sodium, potassium and calcium channels as well as nAChRs (Lebbe et al., 2014). To date, five types of conotoxins, termed $\alpha, \delta, \kappa, \mu$, and $\omega$, have been isolated and characterized, and each type attacks a different target, whereby a-conotoxins mainly inhibit nAChRs (Tsetlin et al., 2009). The a-conotoxins display a consensus fold with a central helical region braced by two conserved disulfide bridges (Azam and Mclntosh, 2009). Based on the number of residues between the second and third, as well as between the third and fourth cysteine residues, $\alpha$-conotoxins are classified into different families, such as $\alpha 3 / 5, \alpha 4 / 3, \alpha 4 / 6$, $\alpha 4 / 7$ et cetera (Mir et al., 2016). As one of the largest and most diverse groups of $\mathrm{nAChR}$ antagonists, they have tremendous therapeutic potential for the treatment of various neurological diseases, including epilepsy and neuropathic pain (Tsetlin et al., 2009; Azam and Mclntosh, 2009).

Owing to their relatively rigid framework structure, combined with great diversity at the amino-acid sequence level, a-conotoxins bind to distinct nAChR subtypes with different selectivity (Rucktooa et al., 2009), which makes them remarkable probes for structural studies. Because ligands that selectively inhibit $\alpha 3 \beta 2, \alpha 6 / \alpha 3 \beta 2 \beta 3$, and $\alpha 3 \beta 4$ nAChRs are lacking, in an earlier study we investigated the $\alpha 4 / 7$ conotoxin LvIA, which is the first a-conotoxin discovered from the carnivorous marine gastropod Conus lividus (Luo et al., 2014). It has high affinity for $\alpha 3 \beta 2 \mathrm{nAChRs}$ with an $\mathrm{IC}_{50}$ of $8.7 \mathrm{nmol} / \mathrm{L}$, and it is notable for being their most selective known probe, as it can distinguish the $\alpha 3 \beta 2$ nAChRs from the $\alpha 6 / \alpha 3 \beta 2 \beta 3\left(\mathrm{IC}_{50} 108 \mathrm{nmol} / \mathrm{L}\right)$ and $\alpha 3 \beta 4$ $\mathrm{nAChR}\left(\mathrm{IC}_{50} 148 \mathrm{nmol} / \mathrm{L}\right)$ subtypes (Luo et al., 2014). As $\alpha 3^{*}$ nAChRs are likely to modulate pain sensation and cardiovascular function, and the a3 subunit is structurally very closely related to a6 (Salas et al., 2009; Paolini and De Biasi, 2011), strategies to selectively distinguish between the $\alpha 3^{*}$ and $\alpha 6^{*}$ subunits, as well as to modulate the function of $\alpha 3^{*}$ $\mathrm{nAChRs}$ are of great importance.

To reveal the mechanism responsible for the distinctive binding profile and selectivity of LvIA towards different $\alpha 3^{*} n A C h R s$, we solved the crystal structure of a-conotoxin LvIA in complex with the acetylcholine binding protein from Aplysia californica (Ac-AChBP) at $3.4 \AA$ resolution. Based on this complex structure, together with homology models based on other nAChR subtypes, as well as binding affinity assays, we offer an explanation for its binding features, which has significant implications for the design of new therapeutic $\alpha$-conotoxin derivatives.

\section{RESULTS}

\section{Overall structure of the Ac-AChBP complex}

We solved the crystal structure of Ac-AChBP in complex with a-CTx LvIA at $3.4 \AA$ resolution, using the molecular replacement method (Table 1). The complex displays a striking structural resemblance to earlier Ac-AChBP/a-CTx structures that were solved before. Upon structural superimposition, the Ac-AChBP/LvIA complex structure had a RMSD of $0.72 \AA$ for all paired $C \alpha$ atoms compared with the Ac-AChBP/PnIA (A10L, D14K) complex (PDB code 2BR8) (Celie et al., 2005), $0.52 \AA$ with the Ac-AChBP/Iml complex (PDB code 2C9T and 2BYP) (Hansen et al., 2005; Ulens et al., 2006), $0.92 \AA$ with the Ac-AChBP/TxIA (A10L) complex (PDB code 2UZ6) (Dutertre et al., 2007), $0.78 \AA$ with the 
Table 1. Crystal diffraction data collection and structural refinement statistics

\begin{tabular}{|c|c|}
\hline Data collection & \\
\hline Beamline & SSRF BL17U \\
\hline Wavelength & $0.9796 \AA$ \\
\hline Space group & $P 2{ }_{1} 2_{1} 2_{1}$ \\
\hline \multicolumn{2}{|l|}{ Cell dimensions } \\
\hline$a, b, c(\AA)$ & $77.39,83.99,209.68$ \\
\hline$\alpha, \beta, \gamma\left({ }^{\circ}\right)$ & $90,90,90$ \\
\hline Resolution $(\AA)$ & $27.71-3.44(3.56-3.44)$ \\
\hline$R_{\text {merge }}(\%)$ & $17.0(87.6)$ \\
\hline //ठI & $8.5(3.0)$ \\
\hline Completeness (\%) & 98 \\
\hline Redundancy & $5.0(5.1)$ \\
\hline \multicolumn{2}{|l|}{ Refinement } \\
\hline Resolution ( $\AA$ ) & $27.71-3.44(3.56-3.44)$ \\
\hline No. reflections & $18405(1463)$ \\
\hline$R_{\text {work }} / R_{\text {free }}(\%)$ & $23.9 / 28.3$ \\
\hline No. atoms & 8833 \\
\hline B-factors $\left(\AA^{2}\right)$ & 94.75 \\
\hline \multicolumn{2}{|l|}{ r.m.s. deviations } \\
\hline Bond lengths $(\AA)$ & 0.004 \\
\hline Bond angles $\left({ }^{\circ}\right)$ & 0.66 \\
\hline \multicolumn{2}{|c|}{ Ramachandran plot (\%) } \\
\hline Favored & 98.45 \\
\hline Allowed & 1.55 \\
\hline Disallowed & 0 \\
\hline
\end{tabular}

Ac-AChBP/BulA complex (PDB code 4EZ1) and $0.34 \AA$ with the Ac-AChBP/GIC complex (PDB code 5CO5) (Lin et al., 2016). The protein forms a windmill-like pentamer along a five-fold axis, forming five highly similar ligand-binding sites between two adjacent protomers (Fig. 1). Upon binding, $a-C T x s$ are buried in the five ligand-binding sites (Fig. 1A). a-CTx LvIA is a C-terminally-amidated peptide comprising 16 amino acids with two disulfide bridges. In the ligandbinding site, it shares a common orientation with other previously determined $\alpha$-CTxs (Fig. S1), with its central helix protruding into the binding site and the $\mathrm{N}$ - and $\mathrm{C}$-termini located at the bottom and top of the binding site, respectively (Fig. 1B)

\section{Structural basis for interactions between the $\alpha-C T x s$ LvIA and Ac-AChBP}

Each $\alpha-C T x$ molecule interacts with two adjacent Ac-AChBP protomers at their interface, forming the principal and complementary binding sides. Most interactions on the principal side were between the peptide and the C-loop (Gln-184 Tyr-
193) of one of the Ac-AChBP protomers. Upon binding to LvIA, the $C$-loop has a significant conformational change that was also observed in the complex structures of Ac-AChBP with other a-CTxs (Fig. S2). Residues His-5, Pro-6, Ala-7, and His-12 of $\alpha-C T x$ LvIA play key roles on the principal binding site. His- 5 forms a hydrogen bond with Tyr-91, Pro-6 undergoes a hydrophobic interaction with Tyr-91 and Trp145, Ala-7 displays extensive hydrophobic interactions with Trp-145, Val-146, Tyr-147, and Tyr-193, while a salt bridge between His-12 of $\alpha-C T x$ LvIA and Glu-191 of Ac-AChBP was also observed. In addition, the Cys-2/Cys-8 disulfide bridge of the peptide was found to be stacked against the vicinal Cys-188/Cys-189 disulfide bond of Ac-AChBP (Fig. 2A).

The complementary binding side is situated at the interface between the LvIA and the $\beta$-sheet of another Ac-AChBP protomer. On the complementary binding side, Ser-4 and Asn- 9 of LVIA form hydrogen bonds with Ser-165 and GIn-55 of Ac-AChBP, respectively. An electronic interaction was also observed between Asp-11 of LvIA and Arg-77 of AcAChBP. Overall, the complementary side does not seem to play a significant role in the interaction between the peptide and the Ac-AChBP (Fig. 2B).

\section{Binding assay of LvIA with Ac-AChBP}

To validate our co-crystal structure and identify residues that confer potency for Ac-AChBP, we conducted surface plasmon resonance (SPR) analysis to study the binding affinities of LVIA and its mutants. The results are summarized in Table 2. The wild-type LvIA and its analogues were produced using solid-phase peptide synthesis. Among the studied mutants, $\mathrm{H} 5 \mathrm{~A}, \mathrm{P} 6 \mathrm{~A}$, and H12A completely lost their binding capacity for Ac-AChBP. The other substitutions, such as Ac-AChBP S4A, exhibited comparatively small decreases of binding affinity. The most notable changes were the improved binding affinities of LvIA (N9A) and LVIA (D11A). LvIA (N9A) bound Ac$\mathrm{AChBP}$ with a $K_{\mathrm{d}}$ value of $82.78 \mathrm{nmol} / \mathrm{L}$, which represents a 1.6-fold higher affinity than native LVIA. LvIA (D11A) had a $K_{d}$ value of $17.48 \mathrm{nmol} / \mathrm{L}$ for $A c-A C h B P$, which means that its potency had increased 7.5 -fold.

Homology modeling of rat $\alpha 3 \beta 2, \alpha 6 \beta 2$, and $\alpha 3 \beta 4$ nAChRs and docking with LvIA

LvIA exhibits a high affinity towards the rat $\alpha 3 \beta 2 \mathrm{nAChR}$ $(8.67 \mathrm{nmol} / \mathrm{L})$, but has a 13 -fold lower affinity towards the rat $\alpha 6 / \alpha 3 \beta 2 \beta 3 \mathrm{nAChRs}(108 \mathrm{nmol} / \mathrm{L})$ and a 17 -fold lower affinity towards the rat $\alpha 3 \beta 4$ subtype $(148 \mathrm{nmol} / \mathrm{L})$. To gain molecular insights into the interactions of $L v \mid A$ with the $\alpha 3 \beta 2, \alpha 6 \beta 2$, and $\alpha 3 \beta 4 \mathrm{nAChR}$ subtypes, homology models of the extracellular ligand binding domain of rat $\alpha 3 \beta 2$, $\alpha 6 \beta 2$, and $\alpha 3 \beta 4$ nAChRs bound to LvIA were constructed using the co-crystal structure of Ac-AChBP/LvIA as template. Homology 

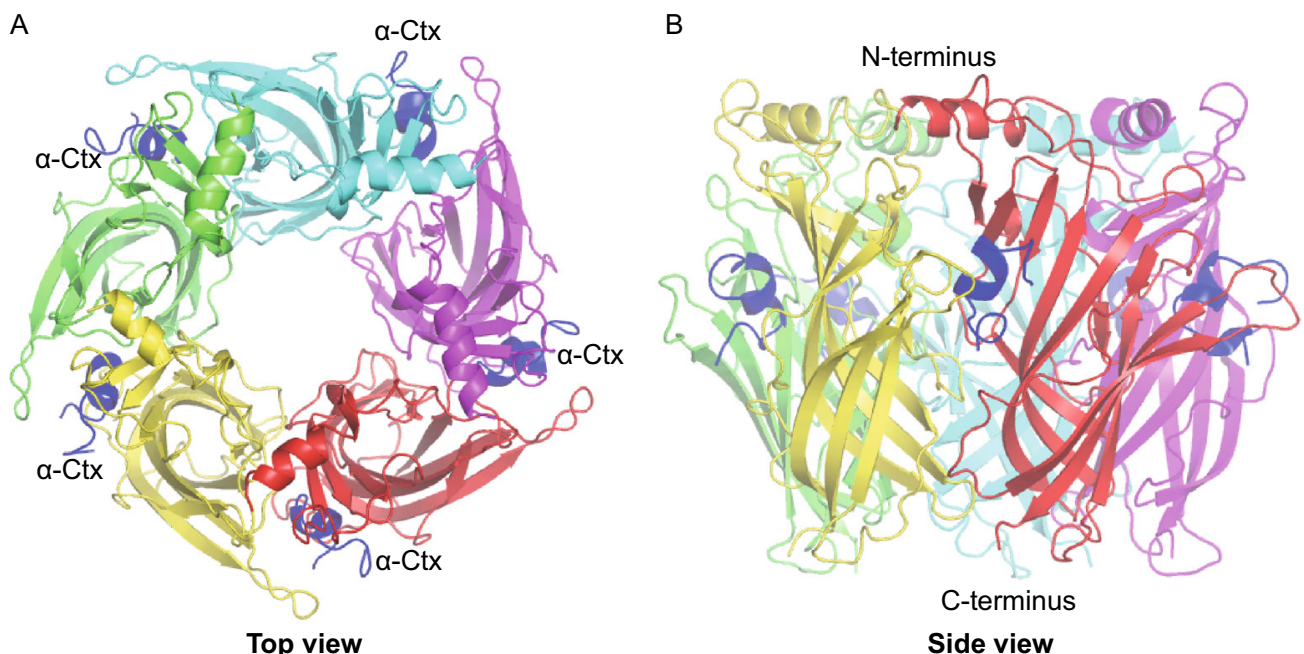

Top view

Side view

Figure 1. The X-ray crystal structure of $\alpha$-conotoxin LvIA binding to Ac-AChBP. (A) The top view of Ac-AChBP/LvIA structure, showing LvIA (blue) in five binding sites. (B) The side view of Ac-AChBP in complex with LvIA, two adjacent protomers of the pentamer with a bound $\alpha$-conotoxin LvIA molecule (in blue).

A

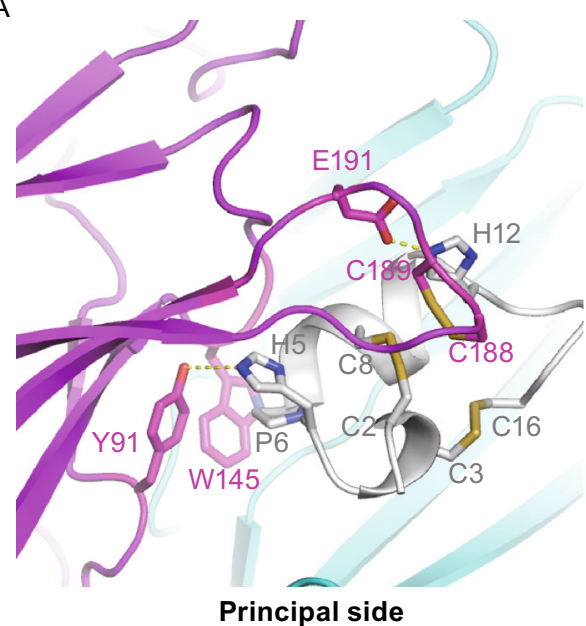

B

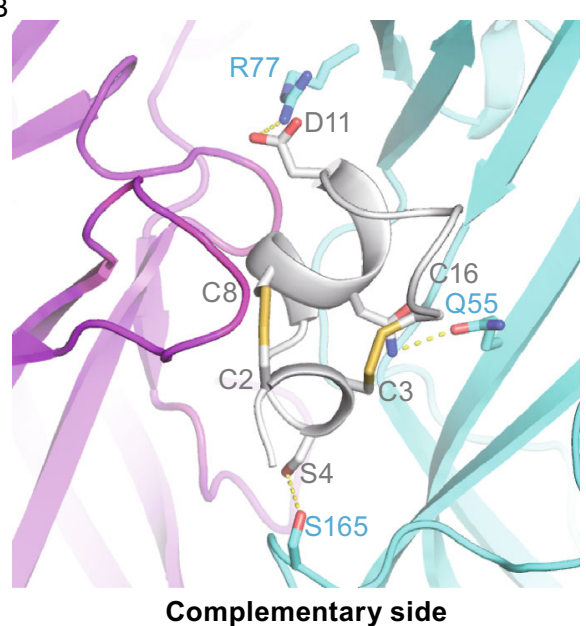

Figure 2. Binding interface between a-conotoxin LvIA and Ac-AChBP. (A) The disulfide bridge packing and hydrogen-bonding interactions (represented by yellow dashed line) on the principal side. Residues His- 5 and His- 12 of the LvIA form hydrogen bonds with Tyr-91 and Glu-191 of the Ac-AChBP, respectively. Disulfide bond C2-C8 in the LvIA closely packed together with C188-C189 in the Ac-AChBP. (B) On the complementary binding side, Ser-4 and Asn-9 of LvIA forms a hydrogen bond (represented by yellow dashed line) with Ser-165 and GIn-55 of Ac-AChBP, respectively. Electronic interaction was also observed between Asp-11 of LvIA and Arg-77 of Ac-AChBP.

modeling has been used in many publications examining conotoxins and nAChRs (McDougal et al., 2013; Luo et al., 2010; Sambasivarao et al., 2014). The interacting residues between LvIA and Ac-AChBP, rat $\alpha 3 \beta 2$, rat $\alpha 6 \beta 2$, and rat a3 $\beta 4 \mathrm{nAChRs}$ are listed in Table 3. A sequence alignment of Ac-AChBP with the rat $\alpha 3, \alpha 6, \beta 2$, and $\beta 4$ nAChRs is shown in Fig. 3. Asterisks $\left(^{*}\right)$ indicate amino acids that are not conserved between the $\alpha 3$ and $\alpha 6$, or $\beta 2$ and $\beta 4$ ligandbinding sites.

In the homology model of LVIA bound to the rat $\alpha 3 \beta 2$ nAChR, we noticed that Pro- 6 of $\alpha-C T x$ LvIA exhibits very strong hydrophobic interactions with Trp-149 of $\alpha 3$ and Trp57 of the $\beta 2$ subunit, on the principal and complementary binding sides, respectively. His- 5 of LvIA has a hydrogen 
Table 2. Binding affinities of $\alpha$-conotoxin LvIA and its mutants towards Ac-AChBP

\begin{tabular}{llll}
\hline Peptide & Sequence & $K_{\mathrm{d}}(\mathrm{nmol} / \mathrm{L})$ & Ratio \\
\hline LvIA WT & GCCSHPACNVDHPEIC & 131.6 & 1 \\
LvIA (S4A) & GCCAHPACNVDHPEIC & 0.18 \\
LVIA (H5A) & GCCSAPACNVDHPEIC & 23.83 & $\mathrm{ND}$ \\
LvIA (P6A) & GCCSHAACNVDHPEIC & $\mathrm{ND}$ & $\mathrm{ND}$ \\
LVIA (A7G) & GCCSHPGCNVDHPEIC* & $\mathrm{ND}$ & $\mathrm{ND}$ \\
LVIA (N9A) & GCCSHPACAVDHPEIC* & $\mathrm{ND}$ & 0.63 \\
LVIA (D11A) & GCCSHPACNVAHPEIC* & 82.78 & 0.13 \\
LvIA (H12A) & GCCSHPACNVDAPEIC & 17.48 & $\mathrm{ND}$ \\
\hline
\end{tabular}

$N D$ not determined.

Asterisks indicate an amidated $\mathrm{C}$ terminusc.

bond with Tyr-193 and extensive hydrophobic interactions with Tyr-93, Tyr-190, and Tyr-197 of the a 3 subunit. Ala-7 exhibits hydrophobic interactions with Trp-149, Tyr-151, and Tyr-197 of the a3 subunit, whereas His-12 of LvIA forms a salt bridge with Glu-195 of the a3 subunit (Fig. 4A and Table 2). These interactions were very similar to the contacts observed in the Ac-AChBP/LvIA crystal structure (Table 2). However, Asn-9 lies within a hydrophobic pocket that is formed by Met-36, Thr-59, and Phe-119 of $\beta 2$ subunit (Fig. 5A), which was different from the hydrogen-bond contact between Ac-AChBP and LVIA.

A comparison of the $\alpha 3 \beta 2$ and $\alpha 6 \beta 2$ nAChR models allowed us to pinpoint the key amino acid residues on the principal side which is responsible for increased binding of LvIA to the $\alpha 3 \beta 2$ vs. the $\alpha 6 \beta 2$ subtype (Fig. 4). One notable change is the substitution of Lys-155 in the a3 subunit with Glu-155 in the a6 subunit. Lys-155 in the a3 subunit, which is a positively charged residue, forms a salt bridge with the negatively charged Asp-11 of LvIA. However, in the a6 subunit, Lys- 155 is replaced by Glu-155, which causes an electrostatic repulsion of the Asp-11 from LvIA. This phenomenon alone may account for a large part of the observed selective potency towards the $\alpha 3 \beta 2$ as opposed to the $\alpha 6 \beta 2$ nAChR subtype.

Additionally, a comparison of the $\alpha 3 \beta 2$ and $\alpha 3 \beta 4 \mathrm{nAChR}$ models allowed us to determine the key residues on the complementary side responsible for increased binding of LvIA to the $\alpha 3 \beta 2$ vs. the $\alpha 3 \beta 4$ subtype (Fig. 5). We previously reported mutational studies conducted to assess the influence of residues from the $\beta 2$ subunit versus those from the $\beta 4$ subunit on the binding of $\alpha-C T x$ LvIA (Zhangsun et al., 2015). Two $\beta 2$ mutations, $\alpha 3 \beta 2$ (F119Q) and $\alpha 3 \beta 2$ (T59K), strongly enhanced the binding affinity of LVIA (IC $\mathrm{C}_{50}$ $0.58 \mathrm{nmol} / \mathrm{L}$ and $I C_{50} 0.96 \mathrm{nmol} / \mathrm{L}$, respectively), and one $\beta 2$ mutation, a3 $\beta 2$ (V111I), substantially reduced the affinity (IC $50126 \mathrm{nmol} / \mathrm{L}$ ) (Zhangsun et al., 2015). According to our a3 $\beta 2$ model, Asn-9 lies within a hydrophobic pocket that is formed by Met-36, Thr-59, and Phe-119, whereas Val-10 undergoes a hydrophobic interaction with Val-111. The side chains of the $\alpha 3 \beta 2$ (T59K) and $\alpha 3 \beta 2$ (F119Q) nAChRs increase the polar contact between LvIA and the $\beta 2$ subunit, forming two hydrogen bonds with Asn-9 of LvIA, which explains the improved binding affinity of LvIA towards the a3ß2 (T59K) and $\alpha 3 \beta 2$ (F119Q) nAChR subtypes. Substitution of valine with isoleucine in the $\alpha 3 \beta 2$ (V111I) nAChR subtype may cause a steric clash between $\mathrm{Val}-10$ and $\beta 2$ $\mathrm{V}-111$, explaining the decreased binding affinity of LVIA. However, in our $\alpha 3 \beta 4$ nAChR model, there is only a small positional shift of Asn-9, making it form a hydrogen bond with Lys-61, but also lose the extensive hydrophobic interactions with Ile-123, Gln-121, and Leu-123. This may explain why LVIA is more potent towards $\alpha 3 \beta 2$, demonstrating that hydrophobic interactions are crucial for the potency of LvIA when acting on the $\alpha 3 \beta 2$ and $\alpha 3 \beta 4 \mathrm{nAChR}$ subtypes.

\section{DISCUSSION}

Analysis of the precise role of $\alpha 3^{*} \mathrm{nAChRs}$ has been hampered by the lack of specific molecular probes. The a-conotoxin LvIA was discovered and characterized in 2014 (Luo et al., 2014). It has a high affinity for the $\alpha 3 \beta 2$ nAChR with an $I_{50}$ of $8.7 \mathrm{nmol} / \mathrm{L}$, and is selective for the $\alpha 3 \beta 2$ nAChR subtype over the $\alpha 3 / \alpha 6 \beta 2 \beta 3$ and $\alpha 3 \beta 4$ subtypes. The previously characterized conotoxins that block $\alpha 3 \beta 2$ nAChRs offer only poor selectivity towards $\alpha 3 \beta 2$ vs. $\alpha 6 \beta 2^{*}$ nAChRs (Luo et al., 2014). However, the expression patterns of $\alpha 3 \beta 2^{*}$ nAChR and $\alpha 6 \beta 2^{*}$ nAChRs overlap in dopaminergic regions, where $\alpha 6 \beta 2^{*}$ nAChRs predominate. LvIA may therefore be a highly valuable probe to interrogate the function and significance of $\alpha 3 \beta 2 \mathrm{nAChRs}$ in normal and disease physiology. In this study, we solved a co-crystal structure of Ac-AChBP/LvIA, and together with LvIA docking on different $n A C h R$ subtypes, concluded that Asn-9 and Asp-11 of $\alpha-C T x$ LvIA are the key residues responsible for its selectivity. According to molecular docking results, the Asp11 residue of LvIA can make a salt bridge with Lys- 155 of the rat $\alpha 3$ subunit, whereas it is electrostatically repulsed by Glu155 of the rat $\alpha 6$ subunit, explaining the large difference in affinity towards the $\alpha 3 \beta 2$ and $\alpha 6 \beta 2 \mathrm{nAChR}$ subtypes. Asn-9 


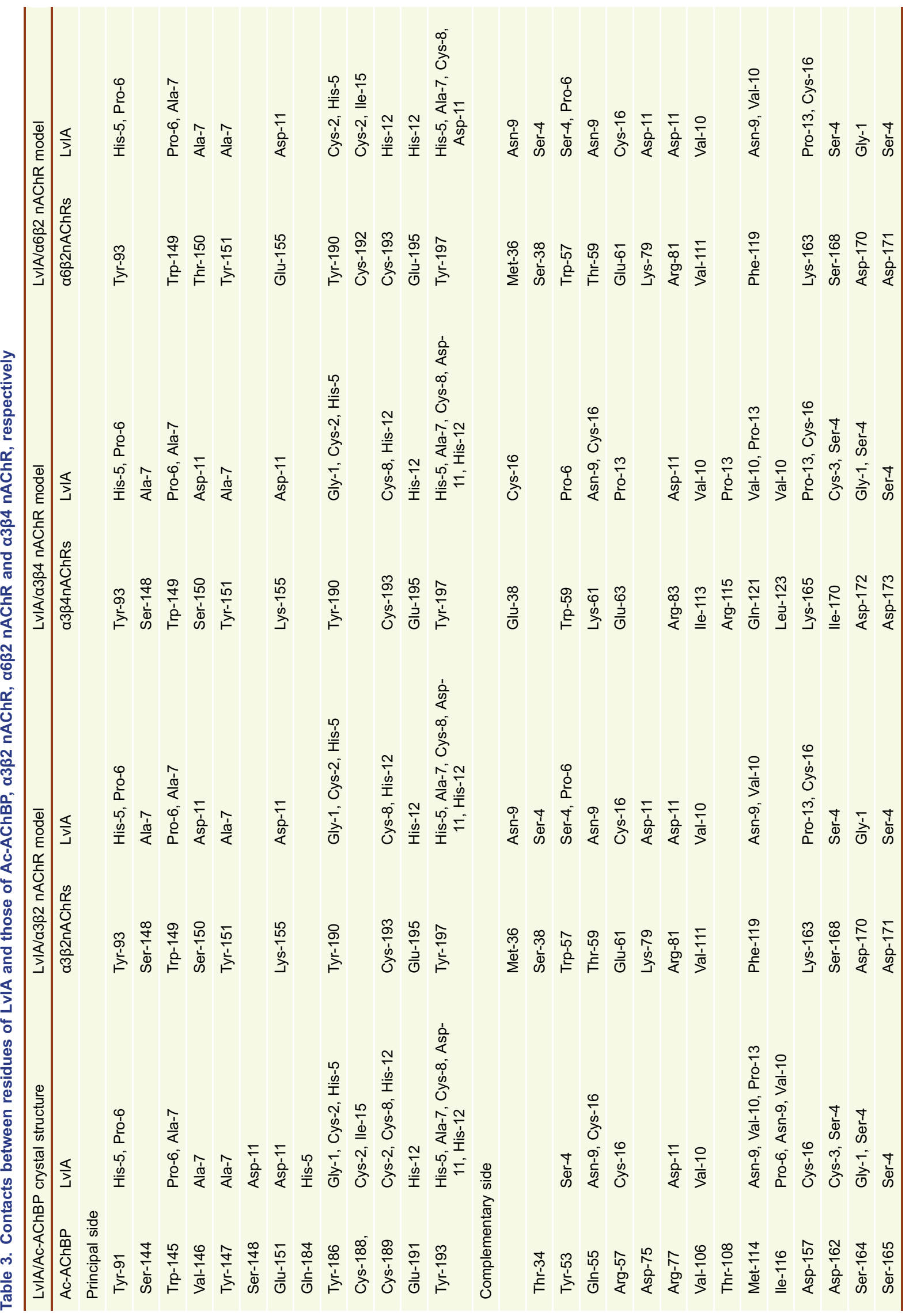




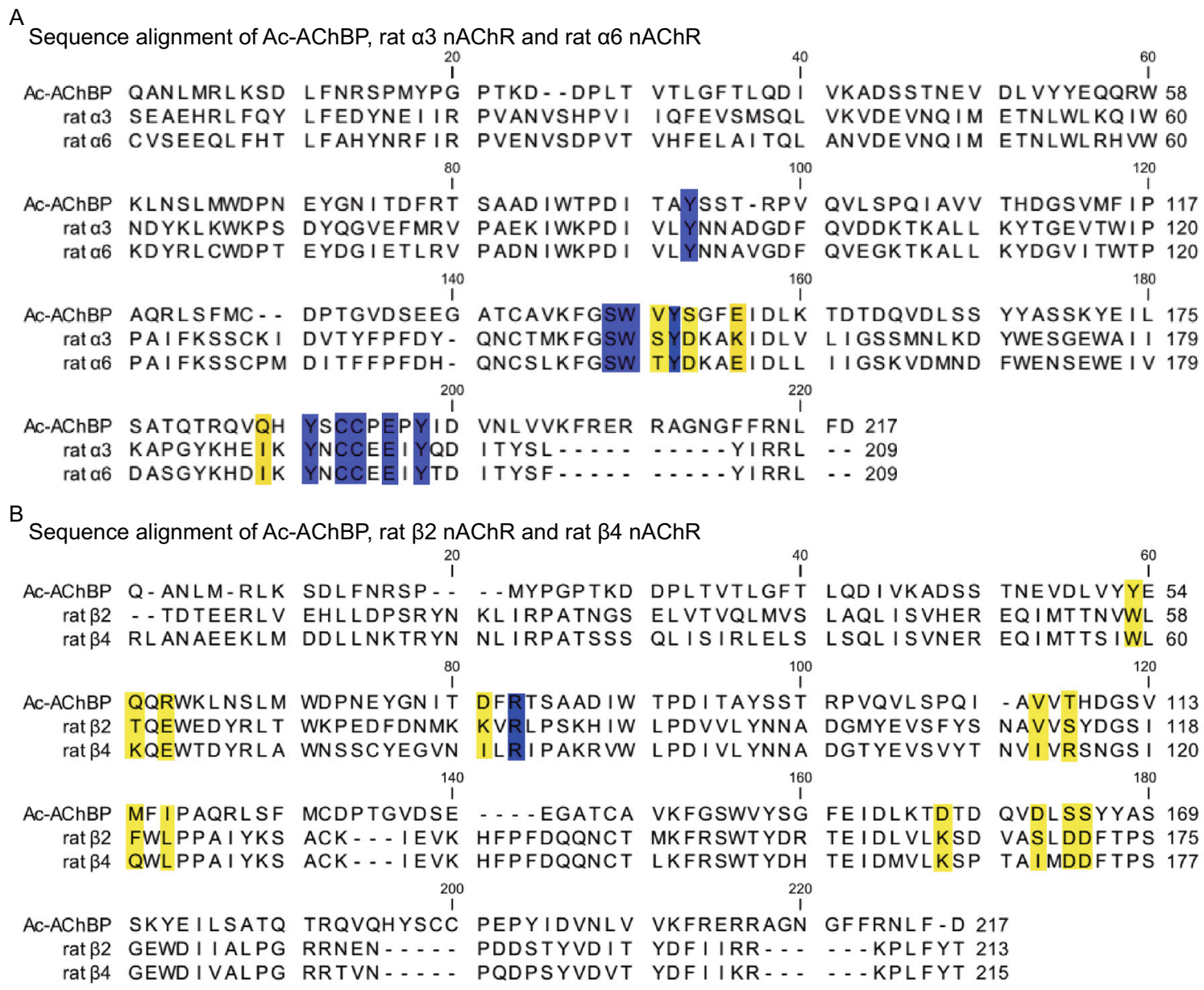

Figure 3. Primary sequence comparison of Ac-AChBP, and the rat $\alpha 3, \alpha 6, \beta 2$, and $\beta 4$ nAChRs. (A) Sequence alignment of Ac$\mathrm{AChBP}$, rat $\alpha 3 \mathrm{nAChR}$, and rat $\alpha 6 \mathrm{nAChR}$; regions colored yellow indicate the amino acids that are not conserved between the $\alpha 3$ and $\alpha 6$ ligand binding sites, regions colored blue indicate the amino acids that are conserved between the Ac-AChBP, $\alpha 3 \mathrm{nAChR}$, and $\alpha 6$ $n A C h R$ ligand binding sites. (B) Sequence alignment of Ac-AChBP, rat $\beta 2 n A C h R s$, and rat $\beta 4 n A C h R s$; regions colored with yellow indicate the amino acids that are not conserved between the $\alpha 3$ and $\alpha 6$ ligand binding sites, regions colored blue indicate the amino acids that are conserved between the Ac-AChBP, $\beta 2 \mathrm{nAChR}$, and $\beta 4 \mathrm{nAChR}$ ligand binding sites.

lies within a hydrophobic pocket that is formed by Met-36, Thr-59, and Phe-119 in the $\alpha 3 \beta 2$ nAChR model, revealing the reason for its more potent selectivity towards the $\alpha 3 \beta 2$ nAChR subtype.

Our results also confirm the results reported by Hone et al. that an N11R substitution in $\alpha-C T x$ PelA essentially abolished the activity of PelA for $\alpha 3 \beta 2$ but not for $\alpha 6 / \alpha 3 \beta 2 \beta 3$ nAChR subtypes (Hone et al., 2013). PelA (N11R) has a positively charged amino acid in the 11th position, while LvIA has a negatively charged residue in its place. Their homology models indicate that PelA(N11R)-binding is disfavored in a3-containing nAChRs, potentially due to a repulsive chargecharge interaction with Lys 152 from the rat $\alpha 3$ subunit (in this publication, Lys152 is equal to Lys155 due to a different numbering system), which was in good agreement with our experimental results. In conclusion, charged residues in the 11th position of the $4 / 7$ a-CTx might be important determinants of binding to $\alpha 6$ and $\alpha 3$ subunits.

Taken together, our findings increase the understanding of the interactions between the $\alpha-C T x$ LvIA and various nAChR subtypes. We identified key residues, such as His-5, Pro-6, Asn-9, Asp-11, and His-12, that are involved in toxinreceptor interaction. This information will be valuable in the design and development of potent $\alpha 3 \beta 2$-selective drugs, with significant implications for the treatment of neuropathic pain and nicotine addiction.

\section{MATERIALS AND METHODS}

Peptide synthesis

We used regio-selective disulfide bond formation with Acm-protected cysteine residues incorporated at positions 1 and 3, and a two-step 
A

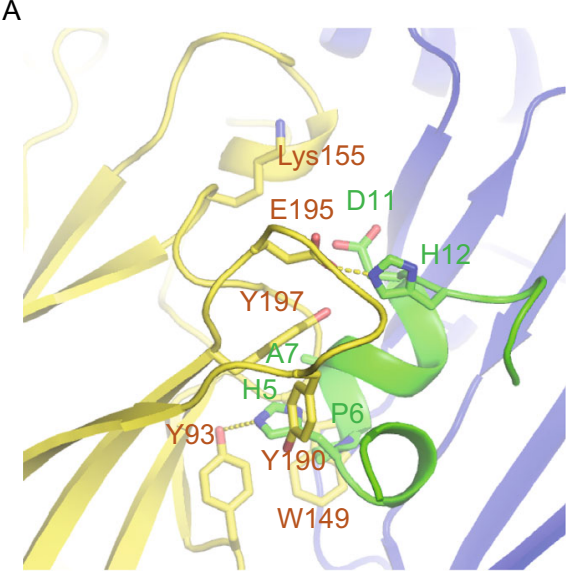

Rat $\alpha 3$ model
B

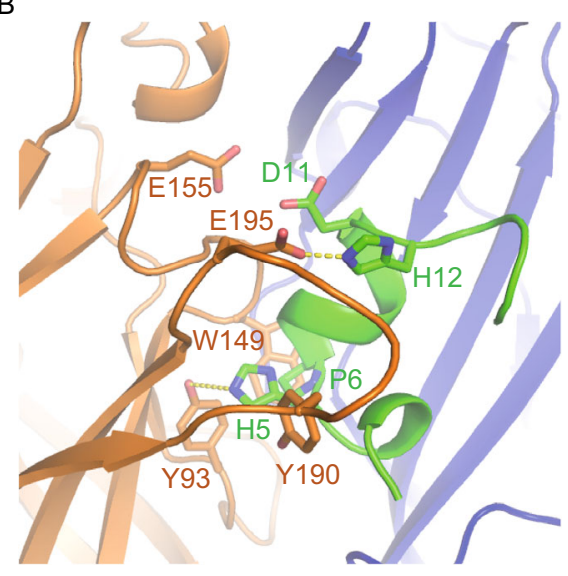

Rat $\alpha 6$ model

Figure 4. Homology modelling of rat $\alpha 3$ and rat $\alpha 6$ bound with $\alpha$-conotoxin LvIA using Ac-AChBP/LvIA as the template. (A) In the rat $\alpha 3$ homology model, the Pro- 6 of $\alpha-C$ Tx LvIA exhibits very strong hydrophobic interactions with the Trp-149 of $\alpha 3$ and Trp-57 of $\beta 2$ subunit both on the principal and complementary binding side; His-5 of LvIA has a hydrogen bond with Tyr-193 and widely hydrophobic interactions with Tyr-93, Tyr-190, and Tyr-197 of a3 subunit; Ala-7 exhibits hydrophobic interactions with Trp-149, Tyr151, and Tyr-197 of $\alpha 3$ subunit and His-12 of LvIA forms a salt bridge with Glu-195 of $\alpha 3$ subunit. (B) In the rat $\alpha 6$ homology model, contacts were very similar with the $\alpha 3$ model except Lys-155 in a3 subunit is replaced by Glu-155 and cause an electrostatic repulsion with Asp-11 of LvIA.

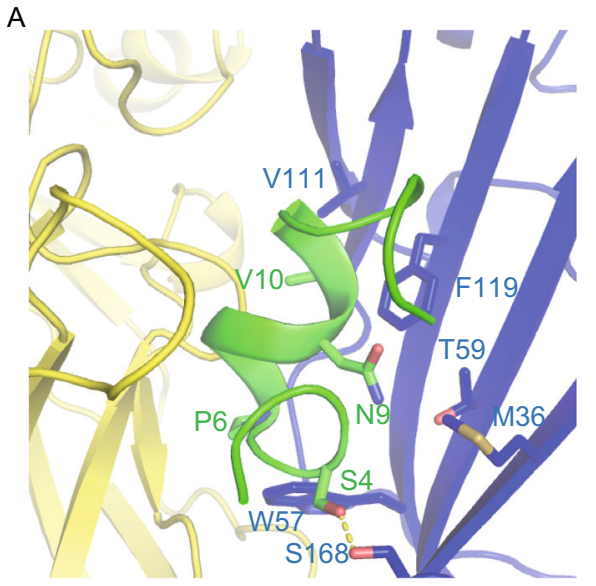

Rat $\beta 2$ model

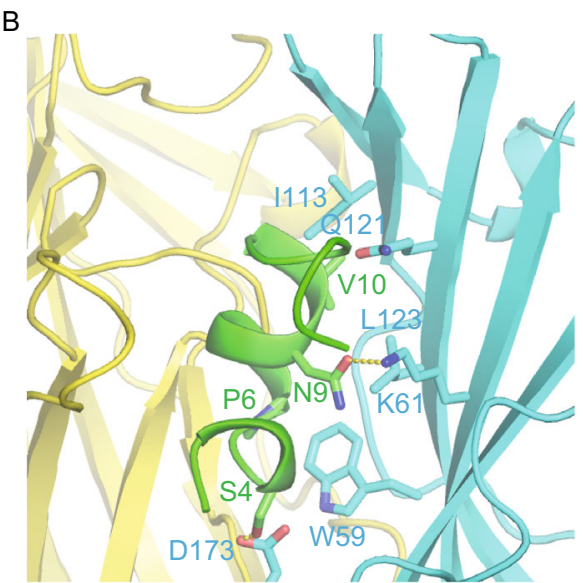

Rat $\beta 4$ model

Figure 5. Homology modelling of rat $\beta 2$ and rat $\beta 4$ bound with $\alpha$-conotoxin LvIA using Ac-AChBP/LvIA as the template. (A) In the rat $\beta 2$ homology model, Asn-9 resides in a hydrophobic pocket that is formed by Met-36, Thr-59, and Phe-119; Val-10 performs a hydrophobic interaction with Val-111. (B) In rat $\beta 4$ homology model, there is a little positional shift of Asn- 9 , making it form a hydrogen bond with Lys-61 but losing extensively hydrophobic interactions with Ile-123, Gln-121, and Leu-123.

oxidation procedure, to produce the alanine mutant peptides in a globular conformation (I-III and II-IV disulfide bonds). Briefly, the first disulfide bridge was closed using $20 \mathrm{mmol} / \mathrm{L}$ potassium ferricyanide in $0.1 \mathrm{~mol} / \mathrm{L}$ Tris- $\mathrm{HCl}, \mathrm{pH} 7.5$. The solution was allowed to react for $45 \mathrm{~min}$, and the monocyclic peptide was purified by reverse-phase HPLC. Simultaneous removal of the acetamidomethyl groups and closure of the second disulfide bridge was accomplished via oxidation, by combining the monocyclic peptide in the HPLC eluent with an equal volume of $10 \mathrm{mmol} / \mathrm{L}$ iodine in $\mathrm{H}_{2} \mathrm{O}$ /trifluoroacetic acid/acetonitrile (78:3:25 by volume) and allowing it to react for $10 \mathrm{~min}$. The reaction was terminated by the addition of ascorbic acid, diluted 20 -fold with $0.1 \%(v / v)$ trifluoroacetic acid, and the bicyclic product purified by reverse-phase HPLC, same as above. The masses of the peptides were verified by matrixassisted laser desorption ionization time-of-flight (MALDI-TOF) mass spectrometry.

Protein expression and purification

Ac-AChBP was overexpressed in High Five insect cells maintained in SIM-HF medium (Sino Biological Inc., China) using the Bac-to-Bac 
baculovirus expression system (Invitrogen, Thermo Fisher Scientific, USA). Sf9 insect cells were maintained in Insect-XPRESS ${ }^{\mathrm{TM}}$ Proteinfree Insect Cell Medium (Lonza, Switzerland). The cDNA encoding the full-length Ac-AChBP was cloned into the pFastBac-Dual vector (Invitrogen) with a C-terminal $6 \times$ His tag to facilitate purification. The plasmid was used to transform competent $E$. coli DH10 Bac cells, and the extracted bacmid was used to transfect Sf9 cells using the Cellfectin II Reagent (Invitrogen). The low-titer virions (P0) were harvested after incubation of the transfected cells at $26^{\circ} \mathrm{C}$ for 7-9 days and amplified to generate high-titer virus stock. An aliquot comprising $10 \mathrm{~mL}$ of the amplified high-titer virus (P1) was used to infect cultures comprising $1 \mathrm{~L}$ of High Five insect cells at a density of $2 \times 10^{6}$ cells $/ \mathrm{mL}$. The culture supernatants containing soluble Ac-AChBP were harvested by centrifugation at $4,000 \mathrm{rpm}$ for $15 \mathrm{~min}$ at $4^{\circ} \mathrm{C}, 48-$ $72 \mathrm{~h}$ after infection. The culture supernatants were further concentrated and buffer-exchanged to HBS (10 mmol/L Hepes, pH 7.2, 150 $\mathrm{mmol} / \mathrm{L} \mathrm{NaCl}$ ) using a $30 \mathrm{KD}$ ultrafiltration cartridge. Ac-AChBP was captured on Nickel-NTA resin (GE Healthcare, USA) and eluted with $500 \mathrm{mmol} / \mathrm{L}$ imidazole in HBS buffer. Further purification was performed by gel-filtration chromatography on the Superdex 200 10/300 High Performance column (GE Healthcare, USA) with protein solution as mobile phase at $0.5 \mathrm{~mL} / \mathrm{min}$.

\section{Crystallization and data collection}

Purified Ac-AChBP and synthesized $\alpha-C T x$ LvIA were mixed at a molar ratio of $1: 1.5$ at $4^{\circ} \mathrm{C}$. After incubation for $2 \mathrm{~h}$, the sample was loaded onto a Superdex 200 10/300 High Performance column (GE Healthcare). The peak fractions were collected and concentrated to $\sim 20 \mathrm{mg} / \mathrm{mL}$ in HBS buffer for crystallization. Crystals were successfully grown at $18^{\circ} \mathrm{C}$ using the sitting drop vapor diffusion method by mixing equal volumes of protein and reservoir solution. Crystals of Ac-AChBP/LvIA grew in buffer containing $1.2 \mathrm{~mol} / \mathrm{L}$ DL-Malic acid $\mathrm{pH} 7.0,0.1 \mathrm{~mol} / \mathrm{L}$ BIS-TRIS propane $\mathrm{pH}$ 7.0. Prior to data collection, the crystals were cryocooled in liquid nitrogen, using reservoir solution plus $20 \%(\mathrm{v} / \mathrm{v})$ glycerol as cryoprotectant. Diffraction data were collected at the BL17U beam line of the Shanghai Synchrotron Research Facility (Shanghai, China). Diffraction data were indexed, integrated and scaled using HKL2000 (Otwinowski and Minor, 1997).

\section{Structure determination and refinement}

The structure was solved via molecular replacement using the PHASER crystallographic software with Ac-AChBP/GIC (PDB code 5CO5) as search model (McCoy et al., 2007). The model was further rebuilt in COOT (Emsley and Cowtan, 2004) and refined in PHENIX (Adams et al., 2002). Structure validation was performed with the program PROCHECK (Laskowski et al., 1993), and all structural figures were generated using PYMOL (http://www.pymol.org/). Data collection and structure refinement statistics are summarized in Table 1.

\section{SPR analysis}

Real-time binding analysis using surface plasmon resonance (SPR) was conducted on a Biacore S200 instrument (GE Healthcare, USA) at $25^{\circ} \mathrm{C}$. Ac-AChBP was immobilized on a research-grade CM5 sensor chip (GE Healthcare, USA) using the amine-coupling method. Flow cell 1 was left blank as a reference. Ac-AChBP $(20 \mu \mathrm{g} / \mathrm{mL})$ in $10 \mathrm{mmol} / \mathrm{L}$ sodium acetate $\mathrm{pH} 5.0$ was immobilized to 600 response units in flow cell 2 . For the collection of data, $\alpha-C T x$ LVIA and its mutants were injected into the flow cells in a buffer comprising $10 \mathrm{mmol} / \mathrm{L}$ HEPES $\mathrm{pH} 7.2,150 \mathrm{mmol} / \mathrm{L} \mathrm{NaCl}$, and $0.005 \%(v / v)$ Tween-20 at various concentration using a $30 \mu \mathrm{L} / \mathrm{min}$ flow rate. Data were analyzed using the Biacore $\$ 200$ evaluation software by fitting to a 1:1 Langmuir binding model.

\section{Homology modelling and docking}

All the modelling and docking were performed in Discovery Studio Client 4.0 (Accelrys, San Diego, CA). The molecular models of extracellular ligand-binding domains of the rat $n A C h R s$ such as $\alpha 3 \beta 2$, $\alpha 6 \beta 2$, and $\alpha 3 \beta 4$ were generated based on the template of AcAChBP structure using the homology modelling program Modeler (Webb, 2014). The LvIA docking was based on the reference model of the Ac-AChBP/LvIA complex. The models were refined with a side-chain refinement and energy minimization process. All modelling and docking structures were verified by the program Profiles$3 \mathrm{D}$ in the Discovery Studio platform, as well as by the MolProbity server (Davis et al., 2007; Davis et al., 2004).

\section{PDB deposition}

The coordinates and diffraction data have been deposited into the Protein Data Bank with accession code 5XGL.

\section{ACKNOWLEDGEMENTS}

We thank scientists at SSRF BL17U beam line for assistance in diffraction data collection. This work was supported by the National Natural Science Foundation of China (Grant Nos. 31470751 and U1405228 to Xinquan Wang) and the Beijing Advanced Innovation Center for Structural Biology. This work was also supported, in part, by the Major International Joint Research Project of National Natural Science Foundation of China (81420108028), and Changjiang Scholars and Innovative Research Teams in Universities Grant (IRT_15R15).

\section{ABBREVIATIONS}

5-HT3, serotonin; a-CTx, a-conotoxin; Ac-AChBP, acetylcholine binding protein; Ach, acetylcholine; AChBPs, acetylcholine-binding proteins; ECD, extracellular domain; GABAA, gamma-aminobutyricacid; nAChRs, nicotinic acetylcholine receptors; pLGIC, pentameric ligand-gated ion channels

\section{COMPLIANCE WITH ETHICS GUIDELINES}

Manyu Xu, Xiaopeng Zhu, Jinfang Yu, Jinpeng Yu, Sulan Luo, and Xinquan Wang declare that they have no conflict of interest. This article does not contain any studies with human or animal subjects performed by the any of the authors.

\section{AUTHOR CONTRIBUTIONS}

M.X. expressed, purified and crystallized the protein. M.X. and J.Y. carried out the SPR analysis. X.Z. and J.Y. synthesized the peptides. 
M.X. collected and processed the diffraction data. X.W. and M.X. carried out the structural determination and refinement. M.X. and $X$. W. wrote the manuscript with help from all authors. X.W. and S.L. designed the research and supervised the project and commented on the manuscript.

\section{OPEN ACCESS}

This article is distributed under the terms of the Creative Commons Attribution 4.0 International License (http://creativecommons.org/ licenses/by/4.0/), which permits unrestricted use, distribution, and reproduction in any medium, provided you give appropriate credit to the original author(s) and the source, provide a link to the Creative Commons license, and indicate if changes were made.

\section{REFERENCES}

Adams PD et al (2002) PHENIX: building new software for automated crystallographic structure determination. Acta Crystallogr Sect D-Biol Crystallogr 58:1948-1954

Azam L, McIntosh JM (2009) Alpha-conotoxins as pharmacological probes of nicotinic acetylcholine receptors. Acta Pharmacol Sin 30(6):771-783

Beroukhim R, Unwin N (1995) Three-dimensional location of the main immunogenic region of the acetylcholine receptor. Neuron 15(2):323-331

Bourne $Y$ et al (2005) Crystal structure of a Cbtx nd antagonistbound s essential interactions between snake $\alpha$-neurotoxins and nicotinic receptors. The EMBO Journal 24(8):1512-1522

Brejc $\mathrm{K}$ et al (2001) Crystal structure of an ACh-binding protein reveals the ligand-binding domain of nicotinic receptors. Nature 411(6835):269-276

Cecchini M, Changeux J-P (2015) The nicotinic acetylcholine receptor and its prokaryotic homologues: Structure, conformational transitions \& allosteric modulation. Neuropharmacology 96 (Part B):137-149

Celie PHN et al (2004) Nicotine and carbamylcholine binding to nicotinic acetylcholine receptors as studied in AChBP crystal structures. Neuron 41(6):907-914

Celie PHN et al (2005) Crystal structure of nicotinic acetylcholine receptor homolog AChBP in complex with an [alpha]-conotoxin PnIA variant. Nat Struct Mol Biol 12(7):582-588

Davis IW et al (2004) MOLPROBITY: structure validation and allatom contact analysis for nucleic acids and their complexes. Nucleic Acids Res 32(Web Server issue):W615-W619

Davis IW et al (2007) MolProbity: all-atom contacts and structure validation for proteins and nucleic acids. Nucleic Acids Res 35 (Web Server issue):W375-W383

Dellisanti CD et al (2007) Crystal structure of the extracellular domain of nAChR alpha1 bound to alpha-bungarotoxin at $1.94 \mathrm{~A}$ resolution. Nat Neurosci 10(8):953-962

Dineley KT, Pandya AA, Yakel JL (2015) Nicotinic ACh receptors as therapeutic targets in CNS disorders. Trends Pharmacol Sci 36 (2):96-108
Dutertre $S$ et al (2007) AChBP-targeted alpha-conotoxin correlates distinct binding orientations with $\mathrm{nAChR}$ subtype selectivity. EMBO J 26(16):3858-3867

Emsley P, Cowtan K (2004) Coot: model-building tools for molecular graphics. Acta Crystallogr Sect D-Biol Crystallogr 60:2126-2132

Hansen SB et al (2005) Structures of Aplysia AChBP complexes with nicotinic agonists and antagonists reveal distinctive binding interfaces and conformations. EMBO J 24(20):3635-3646

Hendrickson L, Guildford M, Tapper A (2013) Neuronal nicotinic acetylcholine receptors: common molecular substrates of nicotine and alcohol dependence. Front Psychiatry. doi:10.3389/fpsyt.2013.00029

Hone AJ et al (2013) Positional scanning mutagenesis of alphaconotoxin PelA identifies critical residues that confer potency and selectivity for alpha6/alpha3beta2beta3 and alpha3beta2 nicotinic acetylcholine receptors. J Biol Chem 288(35):25428-25439

Hurst R, Rollema H, Bertrand D (2013) Nicotinic acetylcholine receptors: from basic science to therapeutics. Pharmacol Ther 137(1):22-54

Karlin A (2002) Emerging structure of the nicotinic acetylcholine receptors. Nat Rev Neurosci 3(2):102-114

Kouvatsos $\mathrm{N}$ et al (2016) Crystal structure of a human neuronal $n A C h R$ extracellular domain in pentameric assembly: Ligandbound alpha2 homopentamer. Proc Natl Acad Sci USA 113 (34):9635-9640

Laskowski RA et al (1993) PROCHECK-a program to check the stereochemical quality of protein structures. J Appl Crystallogr 26:283-291

Laviolette SR, van der Kooy D (2004) The neurobiology of nicotine addiction: bridging the gap from molecules to behaviour. Nat Rev Neurosci 5(1):55-65

Le Novere N, Corringer PJ, Changeux JP (2002) The diversity of subunit composition in nAChRs: evolutionary origins, physiologic and pharmacologic consequences. J Neurobiol 53(4):447-456

Lebbe EK et al (2014) Conotoxins targeting nicotinic acetylcholine receptors: an overview. Mar Drugs 12(5):2970-3004

Li S-X et al (2011) Ligand-binding domain of an [alpha]7-nicotinic receptor chimera and its complex with agonist. Nat Neurosci 14 (10):1253-1259

Lin B et al (2016) From crystal structure of a-conotoxin GIC in complex with Ac-AChBP to molecular determinants of its high selectivity for $\alpha 3 \beta 2 n A C h R$. Scientific Reports 6:22349

Luo $S$ et al (2010) Atypical alpha-conotoxin LtIA from Conus litteratus targets a novel microsite of the alpha3beta2 nicotinic receptor. J Biol Chem 285(16):12355-12366

Luo $S$ et al (2014) A novel alpha4/7-conotoxin LvIA from Conus lividus that selectively blocks alpha3beta2 vs. alpha6/alpha3beta2beta3 nicotinic acetylcholine receptors. FASEB J 28(4):18421853

McCoy AJ et al (2007) Phaser crystallographic software. J Appl Crystallogr 40(Pt 4):658-674

McDougal OM et al (2013) pKa determination of histidine residues in alpha-conotoxin MIl peptides by $1 \mathrm{H}$ NMR and constant $\mathrm{pH}$ molecular dynamics simulation. J Phys Chem B 117(9):2653-2661

Mir R et al (2016) Conotoxins: structure, therapeutic potential and pharmacological applications. Curr Pharm Des 22(5):582-589 
Miyazawa A et al (1999) Nicotinic acetylcholine receptor at $4.6 \AA$ resolution: transverse tunnels in the channel1. J Mol Biol 288 (4):765-786

Morales-Perez CL, Noviello CM, Hibbs RE (2016) X-ray structure of the human alpha4beta2 nicotinic receptor. Nature 538 (7625):411-415

Nemecz A, Taylor P (2011) Creating an alpha7 nicotinic acetylcholine recognition domain from the acetylcholine-binding protein: crystallographic and ligand selectivity analyses. J Biol Chem 286(49):42555-42565

Ortells MO, Lunt GG (1995) Evolutionary history of the ligand-gated ion-channel superfamily of receptors. Trends Neurosci 18 (3):121-127

Otwinowski Z, Minor W (1997) Processing of X-ray diffraction data collected in oscillation mode. Macromol Crystallogr Pt A 276:307-326

Paolini M, De Biasi M (2011) Mechanistic insights into nicotine withdrawal. Biochem Pharmacol 82(8):996-1007

Rucktooa P, Smit AB, Sixma TK (2009) Insight in nAChR subtype selectivity from AChBP crystal structures. Biochem Pharmacol 78 (7):777-787

Salas R et al (2009) Nicotinic receptors in the habenulo-interpeduncular system are necessary for nicotine withdrawal in mice. J Neurosci 29(10):3014-3018

Sambasivarao SV et al (2014) Cover picture: acetylcholine promotes binding of $\alpha$-conotoxin MII at $\alpha 3 \beta 2$ nicotinic acetylcholine receptors (ChemBioChem 3/2014). ChemBioChem 15 (3):413-424
Smit AB et al (2001) A glia-derived acetylcholine-binding protein that modulates synaptic transmission. Nature 411(6835):261-268

Tsetlin V, Utkin Y, Kasheverov I (2009) Polypeptide and peptide toxins, magnifying lenses for binding sites in nicotinic acetylcholine receptors. Biochem Pharmacol 78(7):720-731

Ulens C et al (2006) Structural determinants of selective alphaconotoxin binding to a nicotinic acetylcholine receptor homolog AChBP. Proc Natl Acad Sci USA 103(10):3615-3620

Unwin N (1993) Nicotinic acetylcholine receptor an $9 \AA$ resolution. J Mol Biol 229(4):1101-1124

Unwin N (1995) Acetylcholine receptor channel imaged in the open state. Nature 373(6509):37-43

Unwin N (2005) Refined structure of the nicotinic acetylcholine receptor at $4 \mathrm{~A}$ resolution. J Mol Biol 346(4):967-989

Webb B, Sali A (2014) Comparative protein structure modeling using MODELLER. Curr Protoc Bioinform 47:5.6.1-5.6.32

Zhangsun D et al (2015) Key residues in the nicotinic acetylcholine receptor beta2 subunit contribute to alpha-conotoxin LvIA binding. J Biol Chem 290(15):9855-9862

Zoli M, Pistillo F, Gotti C (2015) Diversity of native nicotinic receptor subtypes in mammalian brain. Neuropharmacology $96(\mathrm{Pt}$ B):302-311

Zouridakis M et al (2014) Crystal structures of free and antagonistbound states of human alpha9 nicotinic receptor extracellular domain. Nat Struct Mol Biol 21(11):976-980 